"abdominal angina"-a central pain reliably precipitated by meals, often prolonged, and unrelieved by antacids. Complete occlusion of the arterial supply will cause gangrene, but the diagnosis may be difficult in those severely ill from other causes.

The arterial anastomoses are least effective at the junction of territory of the superior and inferior mesenteric artery on the left side of the transverse colon, where the marginal artery is poorly developed, ${ }^{2}$ and this is frequently the site of ischaemic disease. ${ }^{1}$ There are three clinically distinct patterns of large bowel ischaemia. ${ }^{1}$ Complete occlusion of the blood supply causes gangrene; chronic ischaemia may lead to stricture formation; and a transient haemorrhagic colitis may occur.

When ischaemic bowel disease is suspected the diagnosis will generally depend on the appropriate radiological investigations. The findings in plain $x$-ray films may be valuable, ${ }^{34}$ but barium enema and even arteriography may be necessary. Ischaemic lesions are not necessarily due to blockage of the arteries: infarction may occur in patients with severe atheroma. ${ }^{4}$ Patients who have a period of low cardiac output after myocardial infarction or after major surgery are especially at risk. ${ }^{4}$ Infarction of the bowel may come on insidiously, but if a patient develops abdominal pain and distension with fever after a period of circulatory failure the diagnosis should be suspected. Further indicators include diarrhoea and rectal bleeding and haemoconcentration with leucocytosis. In making an early diagnosis, ${ }^{46}$ again plain abdominal radiographs are usually helpful: though occasionally the appearances may be normal, usually there are fluid levels and gaseous distension. Gas may be seen in the bowel wall or elsewhere. Barium enema examination may show "thumb-printing" secondary to severe mucosal oedema. Angiography of the superior mesenteric artery will confirm the diagnosis, ${ }^{2}$ with clear evidence of spasm and poor filling of the arterial arcades.

The poor results of surgical management of infarction suggest that efforts must be made to prevent circulatory failure developing in patients at risk. The diagnosis must be considered early when abdominal symptoms and signs occur, and diagnostic radiology performed without delay. Management is still based on laparotomy with a view to surgical resection. The results are disappointing but they might improve if operation could be performed earlier in the disease. Boley et $a l^{6}$ have proposed an aggressive medical regimen of mesenteric catheterisation and papaverine infusion to dilate the arteries, but this approach should be seen as still under evaluation.

\footnotetext{
1 Marston, A, et al, Gut, 1966, 7, 1.

2 Griffiths, J D, Proceedings of the Royal Society of Medicine, 1966, 59, 881.

3 Samuel, E, and Sinclair, D J, Clinical Radiology, 1968, 19, 303.

4 Aldrete, J S, et al, Surgery, Gynecology and Obstetrics, 1977, 144, 371.

5 Demling, R H, Salvatierra, O, and Belzer, F O, Archives of Surgery, 1975, $110,251$.

- Boley, S J, et al, Surgery Annual, 1973, p 355.
}

\section{Sucrose malabsorption}

In the Western world at least half our daily food intake consists of carbohydrate, with starch $(65 \%)$ and sucrose $(25 \%)$ making up the greater part and only small contributions from lactose, fructose, and glycogen. Starch is mainly digested by pancreatic amylase to form units of two and three glucose molecules, but the junctions of its branching chains (the
1:6 $\propto$ links) are not attacked. The next stage of digestion takes place on the brush border of the intestinal epithelium, where the breakdown products of starch, sucrose, and lactose are further hydrolysed by the appropriate enzymes-maltase, isomaltase, sucrase, and lactase. Absorption then follows. Sucrase splits sucrose into glucose and fructose. Isomaltase hydrolyses the 1:6 $\propto$ links, producing a mixture of short-chain glucose units, which are then split by maltase with or without further help from amylase in the lumen.

The recognition of congenital lactose malabsorption was quickly followed by the first clinical description of sucrose malabsorption as a cause of diarrhoea in infants. ${ }^{1}$ Tolerance tests suggested that not only sucrase but also isomaltase were absent in these patients, ${ }^{2}$ and this was confirmed by enzyme assays on jejunal mucosa. ${ }^{3} \mathrm{~A}$ deficiency of more than one enzyme is unusual in an inborn error of metabolism and is an exception to the rule that one enzyme (or polypeptide) is controlled by one gene. As yet there is no explanation for this departure from the general rule. Sucrase-isomaltase deficiency appears to be rare: only some 100 cases have been reported. It occurs in families and is inherited as an autosomal recessive. ${ }^{45}$ More recently it has been shown to be common in Eskimos-an incidence of $10.5 \%$ has been reported from western Greenland, where it may contribute to the endemic malnutrition. ${ }^{6}$

The condition is present from birth but does not declare itself until sucrose is added to the diet. Breast-fed infants should remain well until mixed feeding is started, though the addition of fruit juices may provoke diarrhoea earlier. Bottle-fed infants will suffer from diarrhoea from birth if the milk used contains sucrose. Symptoms are more severe in the young child. The main finding is chronic watery diarrhoea of fluctuating intensity, which may lead to depletion of fluid and electrolytes. Borborygmi, nappy rash, crying spells, and mild steatorrhoea may also be found. Failure to thrive is most severe in the first year of life, when there is relative loss of weight and failure to grow. The condition becomes progressively less serious as the child gets older, when the residual symptoms are some increase in bowel frequency and minor abdominal distension. Eventually most older children tolerate normal amounts of sucrose in the diet.

The simplest diagnostic test in an infant is a switch to a sucrose-free diet, when the diarrhoea will abate if sucrose deficiency is the cause. A sucrose tolerance test will produce a flat blood glucose curve and also result in acid watery diarrhoea. Alternatively, reducing substances may be identified in the stools after sucrose challenge. The test should be performed cautiously in small infants. Absorption of a mixture of glucose and fructose should be normal. Other possible tests include a sucrose barium meal or measurement of breath hydrogen after a sucrose load. The definitive test is jejunal biopsy, which shows absence of sucrose and isomaltase in a histologically normal mucosa. This test should be carried out in all children with longstanding diarrhoea because long-term treatment may have to be given and the other members of the family investigated if the diagnosis is confirmed.

Treatment is by elimination of sucrose from the diet, whereupon symptoms subside within 24-48 hours. Such a modification may be difficult in the young but gets easier as the child gets older, because he becomes more tolerant of lapses in the diet. Restriction of starch intake is usually unnecessary as starch rarely causes symptoms, though excessive amounts should be avoided. Those patients whose 
motions do not return completely to normal on a sucrose-free diet may be offered starch restriction.

Sucrose malabsorption may not be as rare as has been assumed, even outside Greenland. A large paediatric gastroenterology clinic may expect to identify three to four new families every year. ${ }^{9}$ Certainly the diagnosis has been overlooked in children with chronic diarrhoea. In 1973 the delay in diagnosis in six children varied from one to eight years, ${ }^{7}$ and in 1977 another study found delays of up to four years with as many as six inconclusive hospital admissions in one patient. ${ }^{8}$ In view of the modern tendency to give sucrose to very young babies, who are the least able to tolerate it, early diagnosis is important, for treatment leads to an increase in weight and height ${ }^{8}$ that is vital for the child's development in the long run. The condition should not be left for the child to "grow out of." In adults the syndrome does not rate the same importance, but it should be remembered as a possible cause of refractory diarrhoea which may even appear for the first time after puberty. ${ }^{1011}$

Weijers, H A, et al, Acta Paediatrica, 1961, 50, 55.

2 Prader, A, Auricchio, S, and Mürset, G, Schweizerische medizinische Wochenschrift, 1961, 91, 465.

3 Anderson, C M, et al, Pediatrics, 1963, 31, 1003.

- Burgess, E A, et al, Archives of Disease in Childhood, 1964, 39, 431.

Kerry, K R, and Townley, R R W, Australian Paediatric Fournal, 1965, $1,223$.

'McNair, A, et al, British Medical fournal, 1972, 2, 19.

Ament, M E, Perera, D R, and Esther, L J, fournal of Pediatrics, 1973, 83, 721 .

${ }^{8}$ Gudmand-Höyer, E, Krasilnikoff, P A, Scandinavian fournal of Gastroenterology, 1977, 12, 103

9 Anderson, C M, and Burke, V, Paediatric Gastroenterology, p 205. Oxford, Blackwell, 1975.

10 Sonntag, W M, et al, Gastroenterology, 1964, 47, 18.

1 Neale, G, Clark, M, and Levin, B, British Medical fournal, 1965, 2, 1223.

\section{Thymus hormones}

We now have convincing experimental evidence that the thymus secretes hormones ${ }^{1-3}$ elaborated by its epithelial cells. ${ }^{4}$ Thymus grafts restore the immunological function of thymectomised mice even when transplanted in millipore chambers that allow the passage of soluble factors but not intact cells. ${ }^{3}$ Similarly, thymus grafting in immunodeficient children is often succeeded by so rapid an appearance of immunological competence that it is logical to invoke a hormonal effect. ${ }^{5}$ Yet, despite extensive efforts to extract material from the thymus or the blood of various animals, it has proved difficult to isolate and characterise any thymus hormones.

The thymus has multiple functions. Thymus-derived $\mathrm{T}$ lymphocytes initiate both antibody production and cellmediated immunity, and many experiments have aimed to show that thymus extracts restore the reactivity in vivo of immunologically deprived recipients-commonly thymectomised mice or other species. ${ }^{36}$ One example is the ability of thymus extracts to promote host resistance to virus-induced neoplasms, ${ }^{7}$ while such extracts also appear to limit the development of amyloid in mice repeatedly injected with casein, ${ }^{8}$ a process related to impaired $T$ lymphocyte function in susceptible strains. Moreover, extracts of thymus increase such responses as the graft versus host ${ }^{9}$ and the mixed lymphocyte reaction. ${ }^{10}$ By contrast, other lymphocytes constituting one variety of "suppressor cell"11 limit the duration and intensity of immune responses. Possibly thymus extracts may overcome the regulatory action of these cells with the net result that immune reactions are stimulated ${ }^{12}$; but equally probably some crude thymus extracts preferentially stimulate such suppressor cells.

The relation between the thymus and autoimmune disease has long been debated. In mice multiple thymus grafts have been claimed to impair neuromuscular transmission in a manner resembling myasthenia gravis, ${ }^{13}$ suggesting that this disease is caused by the release of thymus hormones in toxic amounts from a thymus damaged by autoimmune inflammation. When secreted in normal amounts these hormones could have a protective function. Two kinds of studies have been carried out in New Zealand mice and other strains which develop autoimmune disease to investigate this suggestion. Firstly, the serum content of thymus hormone has been shown to be deficient in autoimmune mouse strains compared with that of normal strains. ${ }^{1415}$ Secondly, when thymus hormone has been given directly to New Zealand mice ${ }^{16}$ or provided by thymus grafting, ${ }^{17}$ the onset of autoimmune disorders has been delayed or suppressed. Similarly, autoreactivity in nude mice (which congenitally lack a thymus) is suppressed by such manoeuvres. ${ }^{18}$ It is unlikely that such diverse functions are performed by a single hormone, and crude thymus extracts probably contain a variety of active principles. The concept that "thymus hormone" acts as a single stage in the differentiation of the $\mathrm{T}$ lymphocyte is certainly too simple.

A further problem has concerned the bioassays ${ }^{31419}$ available for measuring thymus hormones (since these hormones cannot yet be measured routinely by direct techniques such as radioimmunoassay). These techniques are difficult to standardise, and may give non-specific results. Moreover, purification of the hormone preparations is difficult. Many investigators have used a fraction of crude thymus extract which contains at least 12 major heat-stable polypeptides with molecular weights ranging from 1200 to $14000 .^{20}$ Such preparations probably contain material which will alter the lymphocyte surface entirely non-specifically. ${ }^{21} 22$ Furthermore, since these preparations are certainly immunogenic the neutralising effect of the recipient animals' antibody response makes the interpretation of long-term experiments extremely difficult. So it is encouraging that a nonapeptide of molecular weight about 900 , recently isolated from normal pig blood, has the properties in vitro and in vivo of thymus hormone. ${ }^{23}$ This material has been characterised, and the information should provide the basis for an acceptable assay; and, as it can also be synthesised, ${ }^{23}$ the lengthy and expensive process for isolating the naturally occurring factor may no longer be necessary.

Thymus extracts have been used to treat patients with immune deficiency ${ }^{24}$ and to correct postulated $\mathrm{T}$ lymphocyte defects in such disorders as malignant disease ${ }^{25-27}$ and uraemia. ${ }^{28} \mathrm{~T}$ lymphocyte deficiency in patients with virus infections such as influenza has also been said to be corrected in vitro. ${ }^{29}$ Such claims should not be accepted uncritically: they recall the similar enthusiasm for transfer factor, most of which has evaporated in the wake of properly controlled trials. Even the beneficial effects of thymus hormones in New Zealand mice are not universally accepted. ${ }^{30}$ The thymus plays an important part in regulating immune responses, and elucidating possible hormonal factors is highly relevant to clarifying the underlying mechanisms. Nevertheless, we must understand these better before we introduce thymus hormones into widespread clinical use. The risks attached to such treatment, including the possible activation of retroviruses (oncogenic RNA tumour viruses), ${ }^{31}$ must also be evaluated. 